\title{
A Rabbit Model of Thrombosis on Atherosclerotic Lesions
}

\author{
Atsushi Yamashita and Yujiro Asada \\ Department of Pathology, Faculty of Medicine, University of Miyazaki, 5200 Kihara, Kiyotake, Miyazaki 889-1692, Japan \\ Correspondence should be addressed to Atsushi Yamashita, atsushi@fc.miyazaki-u.ac.jp
}

Received 14 September 2010; Accepted 29 November 2010

Academic Editor: Oreste Gualillo

Copyright ( 2011 A. Yamashita and Y. Asada. This is an open access article distributed under the Creative Commons Attribution License, which permits unrestricted use, distribution, and reproduction in any medium, provided the original work is properly cited.

\begin{abstract}
Thrombus formation on a disrupted atherosclerotic plaque is a key event that leads to atherothrombosis. Because thrombus is induced by chemical or physical injury of normal arteries in most animal models of thrombosis, the mechanisms of thrombogenesis and thrombus growth in atherosclerotic vessels should be investigated in diseased arteries of appropriate models. Pathological findings of human atherothrombosis suggest that tissue factor, an initiator of the coagulation cascade, significantly affects enhanced platelet aggregation and fibrin formation after plaque disruption. We established a rabbit model of atherothrombosis based on human pathology in which differences in thrombus formation between normal and atherosclerotic arteries, factors contributing to thrombus growth, and mechanisms of plaque erosion can be investigated. Emerging transgenic and stem cell technologies should also provide an invaluable rabbit experimental model in the near future.
\end{abstract}

\section{Introduction}

Acute cardiovascular events usually involve thrombus formation at sites of disrupted atherosclerotic plaque, which is currently referred to as atherothrombosis. Although thrombosis is a major complication of atherosclerosis, it does not always result in complete thrombotic occlusion with subsequent acute symptomatic events [1]. Therefore, thrombus growth is critical to the onset of clinical events. Thrombus formation is probably regulated by the thrombogenicity of exposed plaque constituents, local hemorheology, systemic thrombogenicity, and fibrinolytic activity. In fact, the molecular mechanisms of thrombus formation have been identified in mice due to advances in gene targeting technology. However, thrombus is induced by chemically or physically damaging normal arteries in most of these methods. Therefore, little is known about the mechanisms involved in thrombogenesis and thrombus growth in atherosclerotic vessels.

Tissue factor (TF) is a membrane-bound glycoprotein that is expressed or exposed at sites of vascular injury and is essential for hemostasis. As an initiator of the coagulation system, TF acts as a cofactor for circulating factor VIIa, and it starts a series of proteolytic reactions that culminate in the production of the enzyme thrombin, which is the final effecter of the coagulation system. TF is distributed in the adventitia and variably in the media of normal vessels [2]. Active TF has been pathologically detected in atherosclerotic lesions and in platelet-fibrin thrombus formed at disrupted sites. Atherosclerotic lesions are indispensable for studying atherothrombosis. The lipoprotein profiles of rabbits are similar to those of humans but not mice [3], and rabbits on a hyperlipidemic diet are susceptible to atherogenesis. We thus established a rabbit model of atherothrombosis based on human pathology. This paper focuses on human atherothrombosis, a rabbit model, and its pathophysiological significance.

\section{Human Pathology}

2.1. Pathology of Coronary Atherothrombosis. Arterial thrombi were traditionally considered to mainly comprise aggregated platelets because of rapid flow, and the development of platelet-rich thrombi has been regarded as a trigger of atherothrombosis. However, recent evidence indicates that thrombi on disrupted plaques are composed of aggregated platelets and fibrin, erythrocytes, and white blood cells, which are immunopositive for glycoprotein (GP) IIb/IIIa (a platelet integrin), fibrin, glycophorin A (a membrane 
protein expressed on erythrocytes), von Willebrand factor (VWF, a blood adhesion molecule), and CD16 (a marker of neutrophils) [4-6]. GP IIb/IIIa colocalized with VWF, and TF was closely associated with fibrin [5] (Figure 1). These findings suggest that VWF and/or TF contribute to thrombus growth and to obstructive thrombus formation on atherosclerotic lesions, and that the enhanced platelet aggregation and fibrin formation indicate excess thrombin generation mediated by TF.

TF and its procoagulant activities are overexpressed in human atherosclerotic plaques [7, 8], and macrophages and smooth muscle cells (SMCs) in the intima express TF. The activity of TF is more prominent in fatty streaks and atheromatous plaques than in diffuse intimal thickening in the aorta [8]. Thus, atherosclerotic plaque has the potential to initiate the coagulation cascade after plaque disruption, and TF in such plaque is thought to play an important role in thrombus formation after plaque disruption.

The two major morphological features of plaque disruption are rupture and erosion (Figure 2). Plaque rupture is caused by disruption of the fibrous cap, which allows contact between blood and a thrombogenic necrotized core, resulting in thrombus formation. Rupture probably occurs in plaques with a large necrotized lipid core and fibrous caps that are heavily infiltrated by macrophages, lymphocytes, and occasional smooth muscle cells (SMC)s [9]. Plaque disruption and thrombosis in the absence of fibrous cap thinning characterizes plaque erosion. Angiographically, an artery with eroded plaque is less narrowed and the luminal surface is irregular. The morphological characteristics include an abundance of SMCs and proteoglycan matrix, especially versican and hyaluronan, and no necrotic core. Relatively few macrophages and $\mathrm{T}$ cells are features of plaque erosion compared with rupture [9]. The proportions of fibrin and platelets differ in coronary thrombi on ruptured and eroded plaques. Fibrin- and platelet-rich thrombi develop on ruptured and on eroded plaques, respectively, and both $\mathrm{TF}$ and C-reactive protein are more abundant in ruptured and than eroded plaques [4]. These distinct morphological features suggest that different mechanisms are involved in plaque rupture and erosion.

On the other hand, atherosclerotic plaque disruption does not always result in complete thrombotic occlusion with subsequent acute symptomatic events. Clinical studies using angiography and intravascular ultrasound have found that the rupture of several plaques is a frequent complication among patients with coronary atherothrombosis [10]. Disrupted plaques at various stages of healing are occasionally found in autopsy cases with or without coronary atherothrombosis [11]. To evaluate the incidence and morphological characteristics of thrombi and plaque disruption in patients with noncardiac death, Sato et al. [12] examined 102 and 19 hearts from autopsy cases with noncardiac death and from those who died of acute myocardial infarction (AMI). Surprisingly, they found coronary thrombi in $16 \%$ of the cases with noncardiac death, and most of them had developed on eroded plaque. However, the thrombi were too small to affect the coronary lumen (Table 1). The lipid cores were smaller, the fibrous caps were thicker, and
TABLE 1: Incidence of thrombosis in NCD and AMI.

\begin{tabular}{lccc}
\hline & NCD $(n=102)$ & AMI $(n=19)$ & $P$ value \\
\hline Fresh thrombus $(n)$ & $10(10 \%)$ & $14(74 \%)$ & $<.001$ \\
(i) Erosion & $7(7 \%)$ & $4(21 \%)$ & .07 \\
(ii) Rupture & $3(3 \%)$ & $10(53 \%)$ & $<.001$ \\
Old thrombus & $6(6 \%)$ & $5(26 \%)$ & $<.05$ \\
\hline
\end{tabular}

NCD: noncardiac disease; AMI: acute myocardial infarction (from Sato et al. [12] with permission).

luminal narrowing was more modest in disrupted plaques from cases with noncardiac death than with AMI. These findings suggested that the onset of acute coronary events represents the tip of the atherothrombosis iceberg and that regional factors influence coronary thrombus growth after plaque disruption. Thrombus growth is critical to the onset of clinical events because plaque disruption does not always result in complete thrombotic occlusion with subsequent acute symptomatic events.

\subsection{Mechanisms of Plaque Rupture Determined from Human} Pathology. Accumulating evidence supports the notion that inflammation plays a key role in thinning and disruption of the fibrous cap, which results in plaque rupture. The numerous inflammatory cells in rupture-prone atherosclerotic plaques can produce enzymes that degrade the extracellular matrix of the fibrous cap. Macrophages in human atheromas overexpress interstitial collagenases, gelatinases, and elastolytic enzymes. Activated T lymphocytes and macrophages can secrete interferon $\gamma$ (INF- $\gamma)$, which inhibits collagen synthesis and induces the apoptotic death of SMC [13]. Moreover, INF- $\gamma$ can induce interleukin- (IL-) 18, which accelerates inflammation. Interleukin-18 colocalizes with INF- $\gamma$ in macrophages located at the shoulder region, and it is associated with coronary thrombus formation in patients with ischemic heart disease [14]. Interestingly, the important anti-inflammatory cytokine IL-10 is also upregulated in macrophages in atherosclerotic lesions from patients with unstable compared with stable angina [15]. The heterogeneity of macrophages in atherosclerotic plaque could explain these paradoxical findings [16]. This evidence indicates that an imbalance of the inflammatory pathway participates in plaque destabilization that triggers thrombosis in fibrous cap rupture.

Intraplaque hemorrhage might also trigger plaque rupture. The frequency of previous hemorrhages is greater in coronary atherosclerotic lesions with late necrosis and with a thin fibrous cap than in those with early necrosis or intimal thickening [17]. Intraplaque hemorrhage and iron deposition are more prominent in coronary culprit lesions obtained by directional coronary atherectomy from patients with unstable than with stable angina pectoris. The iron deposition correlates with oxidized low-density lipoprotein and thioredoxin, an antioxidant protein, and also associates with thrombus formation [18]. The pathological findings imply relationships among intraplaque hemorrhage, oxidative stress, and plaque instability. However, direct evidence linking intraplaque hemorrhage and plaque instability remains elusive. 

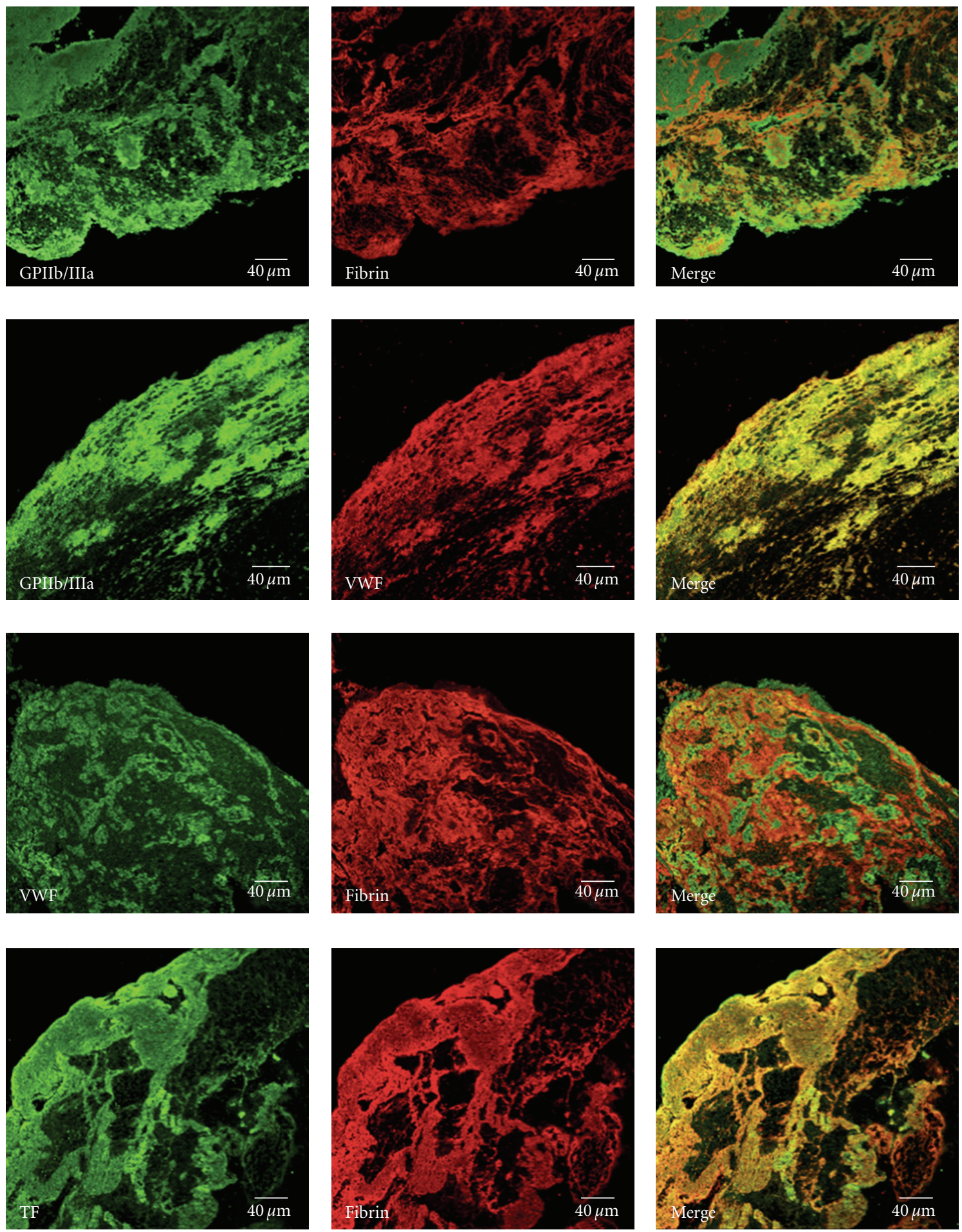

FIGURE 1: Immunofluorescence images of fresh coronary thrombi from patients with acute myocardial infarction. Images of thrombi stained with fluorescein isothiocyanate-labeled glycoprotein (GP) IIb/IIIa, von Willebrand factor (VWF), or tissue factor (TF) (green), Cy3-labeled fibrin or VWF (red), and merged images. Areas with colocalized factors are stained yellow (from Yamashita et al. [5] with permission).

2.3. Mechanism of Plaque Erosion is Unknown. Some authors have postulated that erosion results from vasospasm, but the mechanisms of plaque erosion remain poorly understood. About $80 \%$ of the thrombi associated with plaque erosions are nonocclusive even in sudden coronary death
[9]. Among patients who suddenly die, platelet-rich emboli are found in $74 \%$ and $40 \%$ of those with plaque erosion and plaque rupture, respectively. Because activated platelets release vasoconstrictive agents such as 5-hydroxytryptamine (5-HT, serotonin) and thromboxane A2, these emboli might 

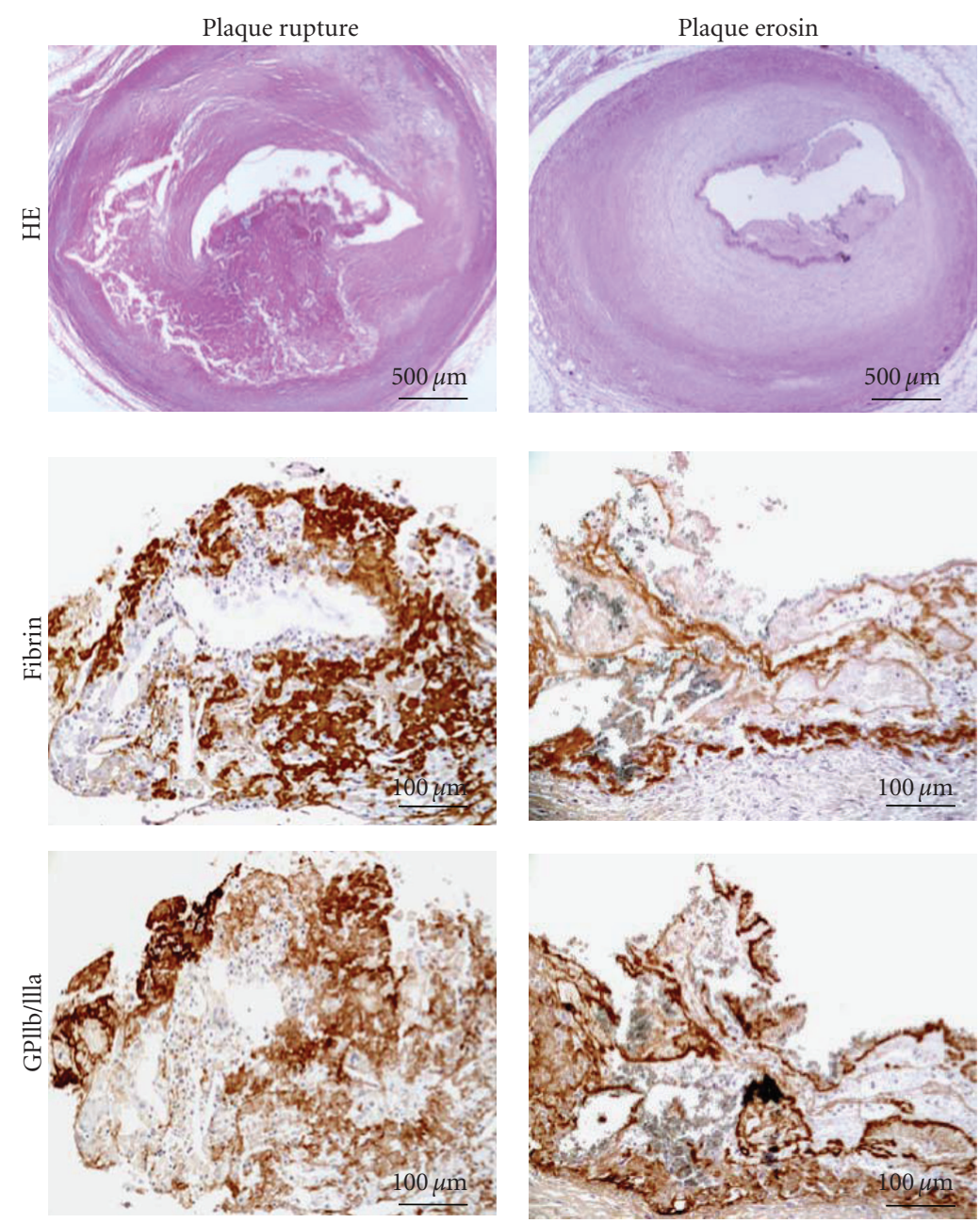

FIGURE 2: Coronary plaque rupture and erosion with thrombi. Large necrotic core and disrupted thin fibrous cap is accompanied by thrombus formation in ruptured plaque. Eroded plaque has superficial injury of SMC-rich atherosclerotic lesion with thrombus formation. Both thrombi comprise platelets and fibrin. HE, hematoxylin eosin stain (from Sato et al. [4] with permission).

increase peripheral resistance leading to altered coronary blood flow. Furthermore, 5-HT can induce vasoconstriction and reduce coronary blood flow in human atherosclerotic vessels, but not in normal arteries [19]. Such vasoconstriction might play an important role in the pathogenesis of plaque erosion. On the other hand, Durand et al. [20] reported that the induction of endothelial apoptosis in vivo leads to both endothelial denudation and vessel thrombosis in rabbit femoral arteries. However, the endogenous triggers of endothelial apoptosis were not determined and the mechanisms of superficial erosive damage to SMCrich plaque remain unknown. However, understanding of these processes can progress using SMC-rich atherosclerotic vessels in rabbits as described below.

\section{Current Arterial Thrombosis Models}

Most established models of thrombosis have used either chemical damage with ferric chloride or rose bengal with focal irradiation. Kurz et al. [21] originally demonstrated that the direct application of ferric chloride solution to the adventitial surface of an artery rapidly induces the formation of a platelet-rich thrombus that typically progresses to complete vascular occlusion. This type of damage produces transmural cell necrosis and disrupts the integrity of the vascular endothelium. However, the mechanism of thrombosis triggered by ferric chloride has not been defined. A small piece of filter paper is saturated with $10 \%-50 \%$ ferric chloride and applied to an artery, where it can be visualized by stereoscopic microscopy. The photochemical model involves the intravenous administration of photoreactive rose bengal to an exposed arterial segment that is then illuminated with green light $(540 \mathrm{~nm})$ delivered from a xenon lamp equipped with a heat-absorbing filter [22]. Rose bengal accumulates in the lipid bilayer of endothelial and other types of cells [23]. Green light triggers a photochemical reaction in rose bengal that produces singlet oxygen and promotes the formation of other reactive oxygen species that damage the endothelium and initiate thrombus formation. The reproducibility of occlusive thrombus formation has 
led to these models becoming the established standard for thrombus model. However, degenerative changes occur in the walls of arteries that have been exposed to ferric chloride and blood content is also altered. The color and shape of these vessels change, the "thrombus" lacks cellular and fibrillar structures, and they microscopically appear rather homogeneous (Figure 3). These findings suggest that denatured materials are a direct consequence of a chemical reaction rather than of sequential biological reactions. Illumination can potentiate platelet function [24], and such thrombus is composed of aggregated platelets [25]. These considerably differ from physiological thrombus formation after plaque disruption. Moreover, many methods involve triggering thrombus formation in normal arteries, which imposes a critical limitation because atherothrombosis in humans always occurs within atherosclerotic arteries. To understand the mechanisms of atherothrombosis requires investigation of a more relevant thrombosis model that comprises diseased arteries.

\section{Rabbit Model of Atherothrombosis}

Pathological findings from humans indicate that TF expression in atherosclerotic plaque plays an important role in thrombus formation after plaque disruption. To understand the role of TF in plaques on thrombus formation and more universally, the mechanisms of atherothrombosis, we used a balloon catheter to induce TF expressing neointima in rabbit femoral artery and to induce thrombus formation on the neointima [26-28].

\subsection{Induction of Macrophage- or SMC-Rich Atherosclerotic} Lesions by Balloon Injury with or without a High-Cholesterol Diet. Rabbits weighting $2.5-3.0 \mathrm{~kg}$ were fed with a conventional or a $0.5 \%$ cholesterol diet for one week before and three weeks after balloon injury to induce SMC- or macrophage-rich neointima in femoral arteries, respectively. The rabbits were anesthetized with pentobarbital $(25 \mathrm{mg} / \mathrm{kg}$, i.v.) and then an angioplasty balloon catheter (diameter, $2.5 \mathrm{~mm}$; length, $10 \mathrm{~mm}$ ) was inserted into the femoral artery under fluoroscopic guidance. The catheter was inflated to $1.5 \mathrm{~atm}$ and retracted by $50 \mathrm{~mm}$ three times to denude the endothelium. This strategy causes SMC- or macrophagerich neointima with increased TF activity to develop several weeks later. Figure 4 shows that neointima developed in femoral arteries three weeks after balloon injury in rabbits fed with conventional or $0.5 \%$ cholesterol diets. The neointimal area was larger in rabbits fed with the cholesterol, than with the conventional diet. Immunohistochemical staining revealed that neointima induced under conventional or cholesterol diet is SMC or macrophage rich, respectively, and that TF was localized in both neointima and adventitia. The activity of TF was assessed as rabbit plasma clotting time increased more in the SMC- and macrophage-rich neointima/media than in normal intima/media $[27,28]$. The increased neointimal TF expression and activity simulated the situation in human atherosclerotic plaques $[7,8]$, and the SMC- or macrophage-rich neointima mimics human

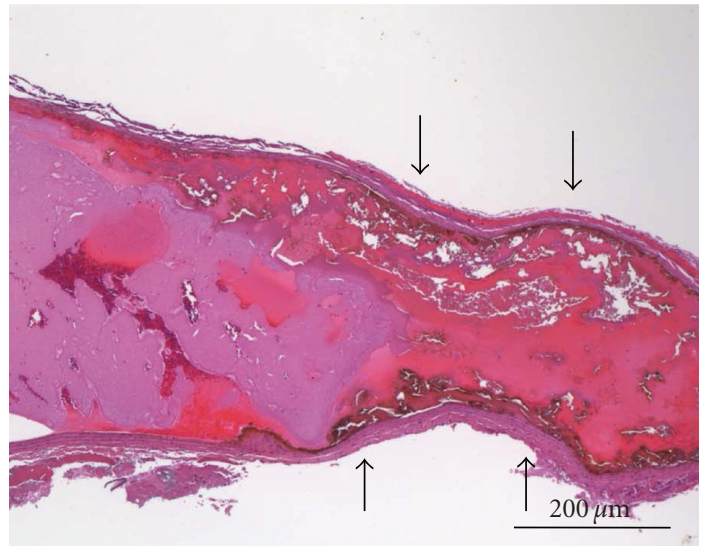

(a)

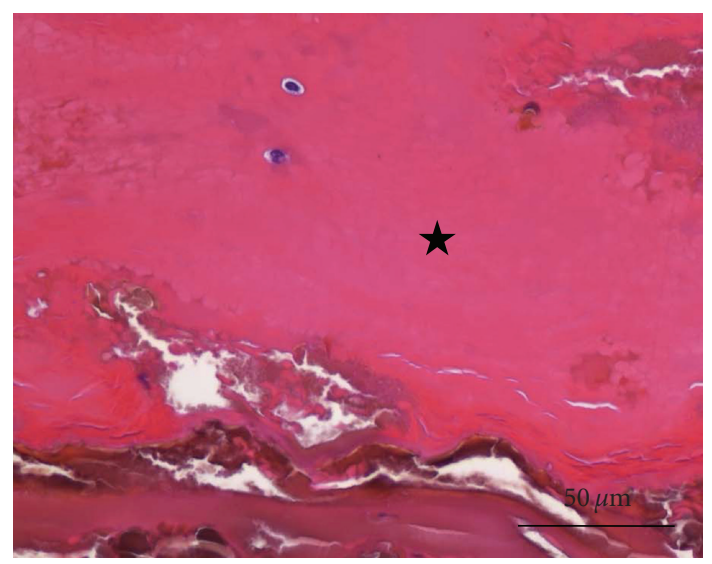

(b)

Figure 3: Histological images of carotid artery from rat ferric chloride model. (a) Longitudinal section of rat carotid artery 20 minutes after initiation of $20 \%$ ferric chloride injury. Arterial wall and blood show marked degenerative or denaturation changes at site of injury (arrows), wall constriction, and brown color at bloodvessel interface. Hematoxylin eosin stain. (b) Higher magnification of attached thrombus $(*)$ shows absence of cellular and fibrillar structures and rather homogeneous appearance. Hematoxylin eosin stain.

fibrocellular and lipid-rich types of plaque, respectively [4, $29,30]$.

However, the remodeling response after balloon injury significantly differs between conventional and cholesterol diets. The numbers of inflammatory cells, especially macrophages, as well as microvessels and matrix metalloproteinase expression increased, and matrix density decreased in the damaged arteries of rabbits fed with a cholesterol diet, resulting in extensive arterial remodeling [31]. These findings indicate that hyperlipidemia directly contributes to arterial remodeling via inflammatory responses after arterial injury.

4.2. Different Mechanism of Thrombus Formation in Atherosclerotic or Normal Artery. The arteries were further damaged using a balloon catheter at three weeks after the first balloon injury to induce thrombus formation on the 

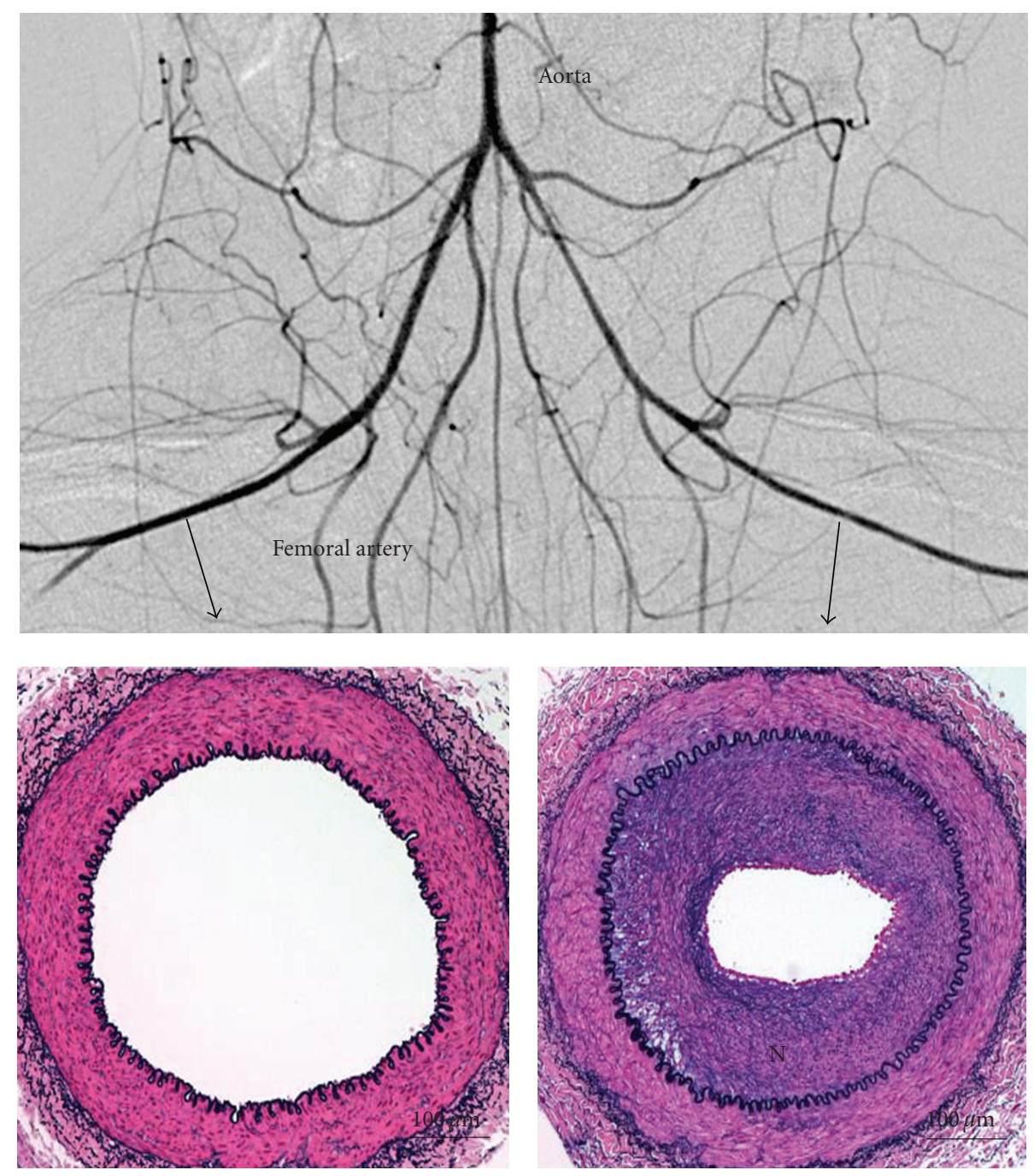

(a)

3 weeks artery balloon injury
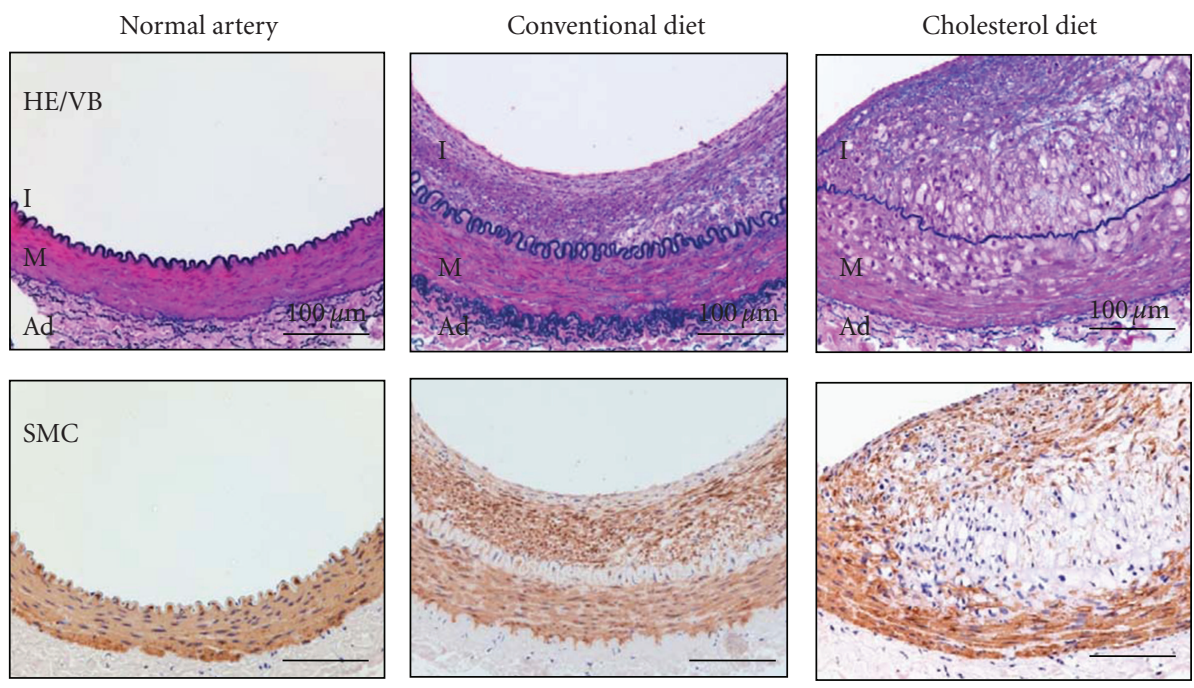

(b)

FIgURe 4: Continued. 

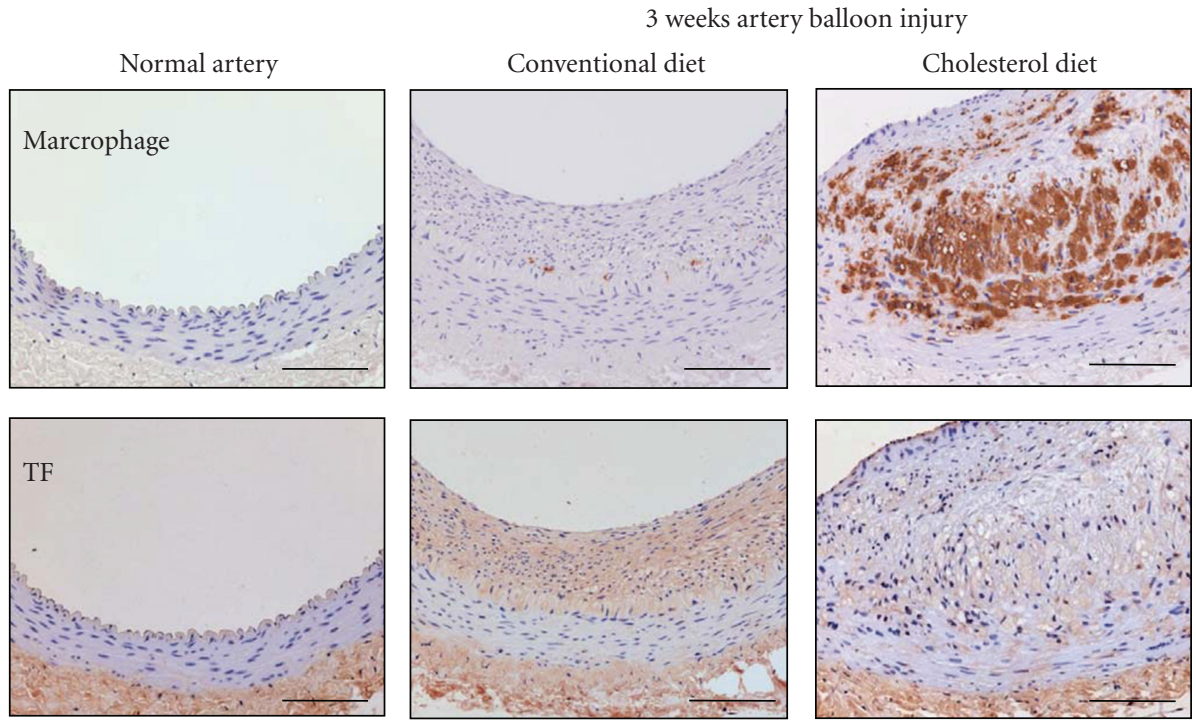

(b)

Figure 4: Angiographic and histological images of rabbit femoral arteries. (a) Representative angiographic and histological image 3 weeks after the balloon injury of left femoral artery. Injured femoral artery shows luminal narrowing and neointimal formation (N). Hematoxylin eosin/Victria blue stain (from Yamashita et al. [27] with permission). (b) Representative immunohistochemical microphotographs of normal and balloon-injured femoral arteries at 3 weeks after injury under conventional or $0.5 \%$ cholesterol diet. Tissue factor (TF) is expressed in neointimal smooth muscle cells (SMCs), macrophages, and adventitia. I, intima; M, media; Ad, adventitia. HE/VB, hematoxylin eosin/victoria blue stain.

neointima. A 2F balloon catheter (Baxter Healthcare) was inserted via the anterior tibial artery into the femoral artery, inflated to $1.4 \mathrm{~atm}$, and retracted three times. Fifteen minutes later, SMC- and macrophage-rich neointima produced large thrombi composed of platelets and fibrin, whereas small platelet thrombi developed on the normal intima of rabbits fed with both conventional and cholesterol diets (Figure 5) [28]. Thrombus formation was assessed as cross-sectional size under a microscope, areas that were immunopositive for GPIIb/IIIa and fibrin, or by blood flowmeter $[27,28]$.

Recombinant tissue factor pathway inhibitor and argatroban, a thrombin inhibitor, significantly reduced platelet aggregation, fibrin formation, and thrombus formation on both SMC- and macrophage-rich neointima, but not on normal intima. These results suggested that the mechanism of thrombus formation on normal and diseased arteries is independent and dependent, respectively, on thrombin [28]. Thrombin is a multifunctional enzyme that is generated on activated platelets by prothrombinase complexes comprising activated factor X (FXa) and factor V. Thrombin promotes the coagulation cascade, activates platelets, and inhibits proteolysis [32]. Our findings indicate that thrombin generation mediated by intimal TF contributes to the formation of large thrombus in atherosclerotic vessels via enhanced platelet aggregation and fibrin formation. In addition, thrombus formation on macrophage-rich neointima was sensitive to inhibitors of TF-activated factor VII (FVIIa), FXa, and thrombin. These results support the notion that thrombus formation in this model is initiated by activation of the TFFVIIa pathway [33].
4.3. Other Atherothrombosis Models. Apolipoprotein E (apoE) is a class of apolipoprotein that is essential for the normal catabolism of triglyceride-rich lipoprotein constituents. Apo E knockout mice develop hypercholesterolemia and atherosclerotic lesions throughout the vasculature with a predilection for bifurcation sites [34]. Eitzman et al. [35] demonstrated that acute hyperlipidemia enhances platelet activation and photochemically induced thrombus formation on carotid atherosclerotic lesions of 30-week-old apoE $\mathrm{E}^{-/-}$mice. They also found shortened occlusion times in 30- versus 6-week-old mice, indicating that thrombogenicity is increased in carotid plaque. Although the mechanism of the enhanced thrombogenicity is unclear, this model is more reflective of atherothrombosis than photochemically induced thrombosis in normal animal arteries. Intravital microscopy has recently allowed real-time imaging of thrombus growth and dissolution. Kuijpers et al. [36] generated a model of thrombus formation evoked by plaque rupture in the carotid arteries of apoE ${ }^{-/-}$mice fed with a western-type diet for 1820 weeks. Localized rupture at carotid plaque was induced by ultrasound, and thrombus formation was visualized by two-photon laser scanning microscopy. Such plaque damage resulted in endothelial denudation, collagen exposure, and mural thrombus formation, which relied on both platelet activation and thrombin generation. This model might prove useful for understanding the molecular mechanisms of dynamic thrombus formation on atherosclerotic lesions.

Constantinides and Chakravarti introduced a rabbit model of atherothrombosis over 30 years ago [37]. Atherosclerotic lesions were induced in the aorta by a cholesterol diet for 2-8 months with or without balloon injury, and 
injected Russell's viper venom and histamine induced the initiation of thrombus on the atherosclerotic lesions. The rate of plaque disruption with thrombus formation was originally $30 \%$ (cholesterol diet) and up to 70\% (cholesterol diet plus initial balloon injury) [38]. Large thrombus in the aorta is useful for investigations using modalities such as magnetic resonance imaging and positron emission tomography $[39,40]$.

\section{Applications for SMC-Rich Atherosclerotic Lesion on Thrombosis}

Intima that is rich in SMCs and extracellular matrix is a basic morphological feature of atherosclerotic lesions [9]. Coronary arteries with SMC-rich intima are susceptible to vasoconstriction or vasospasm induced by some thrombogenic agents [41]. Pathological studies have demonstrated that the coronary arteries of patients with coronary vasospasm have SMC-rich intima [29] and that the intima of eroded plaques contains significantly more SMCs than that of stable or ruptured plaques $[30,42]$. The clinicopathological evidence suggests a relationship between SMC-rich atherosclerotic lesions and thrombogenic vasoconstriction and/or plaque erosion.

5.1. Mechanisms of Plaque Erosion Determined in a Rabbit Model. Unlike those of plaque rupture, the mechanisms of plaque erosion remain obscure. Endothelial cells preferentially undergo apoptosis at areas downstream of atherosclerotic plaques, where blood flow is disrupted and shear stress is lower than at upstream areas [43]. Experimental aortic stenosis can induce acute endothelial changes or damage the normal aorta [44]. Therefore, hemodynamic force, particularly disturbed blood flow induced by stenosis or vasoconstriction, could be a crucial factor in generating surface vascular damage and thrombosis. In fact, blood flow disturbed by acute vascular narrowing induced superficial erosive injury to SMC-rich neointima at poststenotic regions in rabbit femoral arteries. The blood flow of the femoral arteries was reduced to $75 \%$ with a vascular occluder. The vascular narrowing induced a disturbed blood flow at poststenotic portion. Figure 6(a) shows microscope images of a longitudinal section of neointima from the poststenotic region at $15 \mathrm{~min}$ after vascular narrowing. The endothelial cells and SMCs at this region were broadly detached and associated with platelet adhesion to the subendothelium (Figures 6(a) and 6(b)). The superficial erosive injury also induced the apoptosis of endothelial cells and superficial SMCs within 15 minutes [45]. The neointima were completely lined by endothelial cells in the absence of vascular narrowing, and there were no apoptotic cells in the intima. Thus, disturbed blood flow can induce superficial erosive injury to SMC-rich plaque and thrombus formation at the poststenotic region. Computational fluid simulation analysis has indicated that oscillatory shear stress contributes to the progress of erosive damage to the neointima [45]. Although direct clinical evidence has not yet supported the notion that coronary artery vasospasm plays a role in plaque erosion, the superficial erosive injury of SMC-rich neointima induced by disturbed blood flow is similar to that of human plaque erosion $[4,9]$. In addition, platelets and blood coagulation are activated in the coronary circulation after vasospastic angina [46]. Therefore, these evidences together suggest that acute-onset disturbed blood flow caused by vasoconstriction could trigger plaque erosion. Hemodynamic factors could thus play important roles in the process of plaque erosion.

5.2. SMC-Rich Atherosclerotic Lesions on Thrombogenic Vasoconstriction. Coronary artery contraction and spasm at sites of atherosclerotic lesions have been studied in detail [47], whereas relatively little is understood about the thrombusmediated constriction of diseased arteries. Plaque disruption activates platelets that release many vasoactive substances into the circulation. Rabbit femoral arteries with SMC-rich atherosclerotic lesions developed a hypercontractile response to $5-\mathrm{HT}$ via $5-\mathrm{HT}_{2 \mathrm{~A}}$ receptors, but not to any of adenosine diphosphate, adenosine triphosphate, or thrombin. Notably, 5-HT caused the SMC-rich atherosclerotic lesion itself to contraction. Moreover, the hypervasoconstriction of SMCrich atherosclerotic vessels alters blood flow during thrombogenesis and affects the formation of occlusive thrombus in rabbits $[48,49]$. The results indicate that thrombogenic vasoconstriction induces luminal stenosis with reduced blood flow in culprit vessels and promotes further thrombus growth.

\section{Applications for Thrombus Growth Study}

As described above, plaque disruption does not always result in the complete thrombotic occlusion and thrombus growth that are critical to the onset of clinical events. Thrombus formation is probably regulated by vascular wall thrombogenicity, local blood flow, and blood content. However, their contribution to thrombus growth has not yet been defined.

6.1. Role of Coagulation Factors on Thrombus Growth. The most fundamental difference between normal and atherosclerotic arteries is that active TF is abundant in atherosclerotic lesions [7, 8]. Therefore, vascular wall TF might contribute to thrombus formation/growth on such lesions. However, recent studies have detected low levels of TF in the blood that are nevertheless sufficient to support clot formation in vitro. Plasma TF levels are elevated in patients with unstable angina and AMI, and they correlate with adverse outcomes [50]. Therefore, whether or not TF derived from the vascular wall and/or blood supports thrombus propagation remains controversial. Hematopoietic cell-derived, TF-positive microparticles contribute to laser injury-induced thrombosis in the microvasculature of the mouse cremaster muscle [51]. In contrast, TF derived from hematopoietic cells does not contribute to photochemicallyinduced thrombosis in the mouse carotid artery [52]. The atherosclerotic lesions in rabbit femoral arteries expressed TF and increased procoagulant activity compared with normal femoral arteries. Thrombin-dependent large platelet-fibrin thrombi were induced in the neointima damaged by balloon 


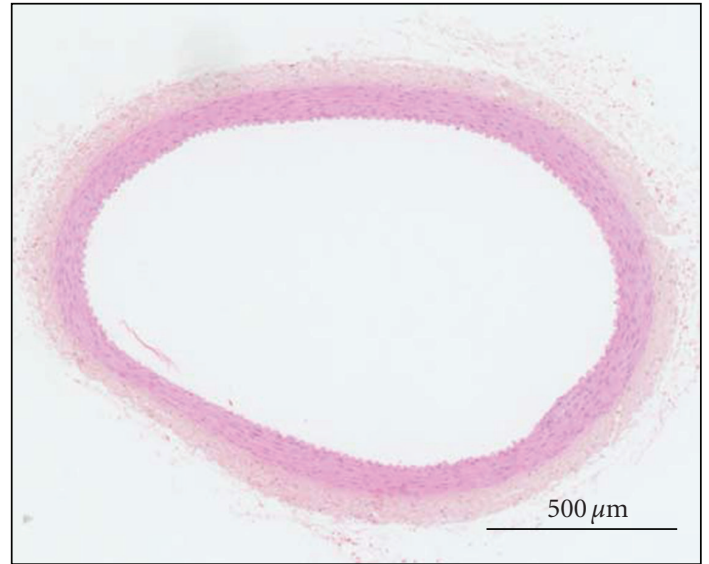

(a)
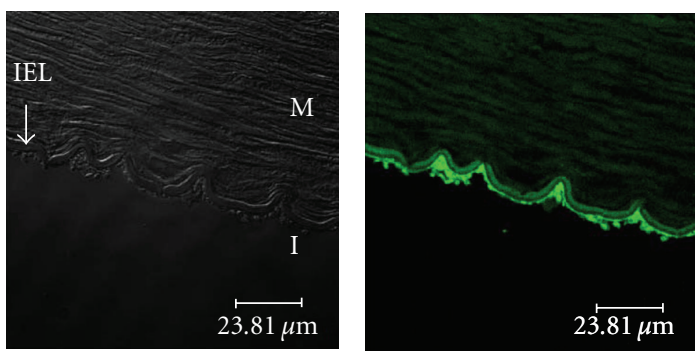

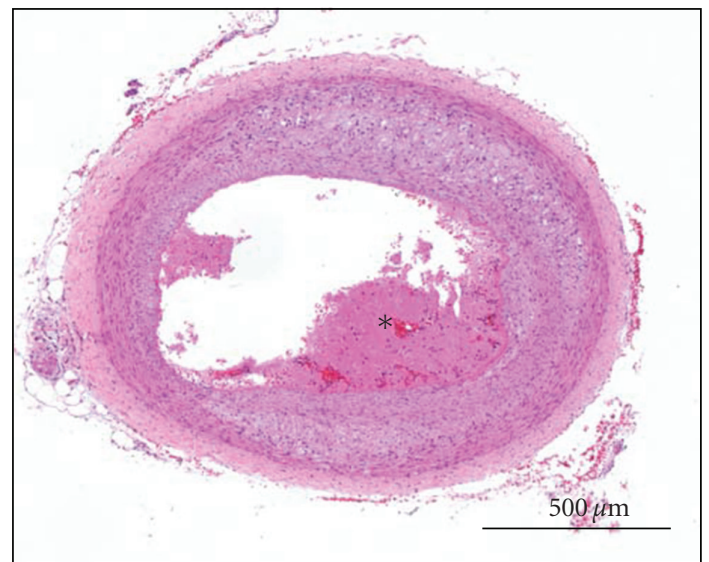

(b)
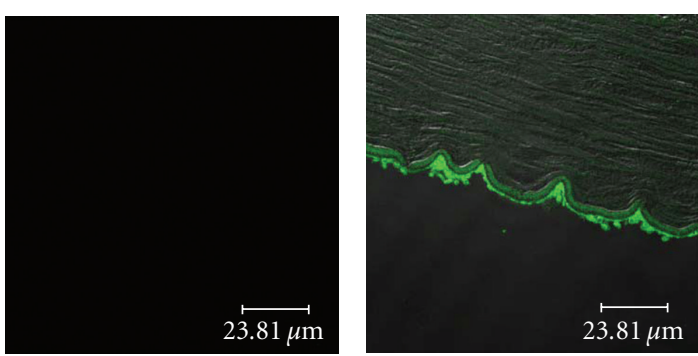

(c)
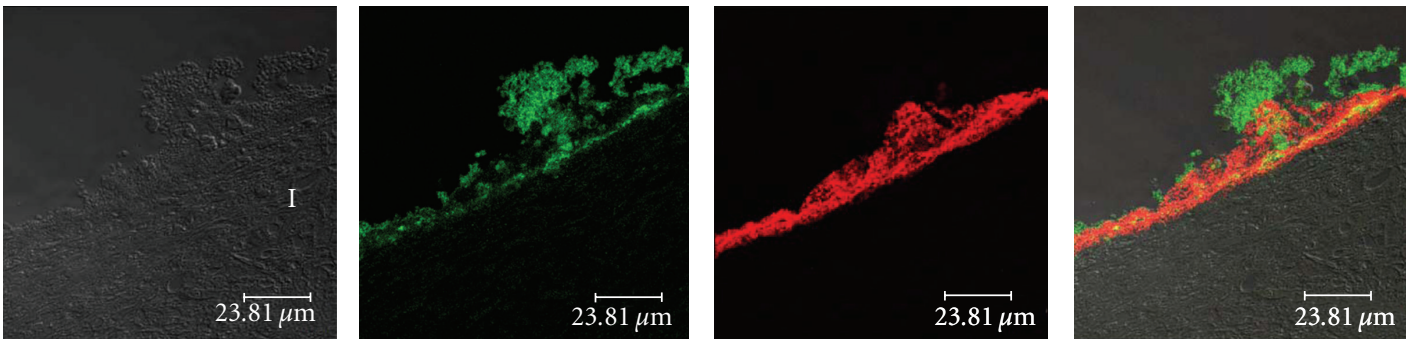

(d)

FIGURE 5: Light and immunofluorescence microphotographs of thrombus on rabbit femoral arteries. Representative light and immunofluorescence microphotographs of thrombi that developed in normal femoral artery 15 minutes after balloon injury and in atherosclerotic neointima from rabbits on $0.5 \%$ cholesterol diet. Whole images of arterial sections of normal artery (a) or atherosclerotic artery (b) 15 minutes after balloon injury. The size of thrombus significantly differs between normal artery thrombus and atherothrombus $(*)$. The normal artery thrombus is too small to recognize. Hematoxylin eosin stain. Rows (c, d) show differential interference contrast, fluorescein isothiocyanate-labeled GPIIb/IIIa (green), Cy3-labeled fibrin (red), and merged immunofluorescent images. Areas with colocalized factors are stained yellow. Thrombi on normal intima are composed of small aggregated platelets (c), whereas those on atherosclerotic neointima comprise platelets and fibrin (d). I, intima; M, media; IEL, internal elastic lamina (from Yamashita et al. [28] with permission).

denudation, whereas thrombin-independent small platelet thrombi were induced in normal femoral arteries damaged in the same manner (Figure 5) [27, 28]. Moreover, whole blood coagulation in the rabbit model of atherothrombosis was not affected by inhibiting blood TF using a TF antibody even under hyperlipidemic conditions [28]. Therefore, atherosclerotic plaque-derived TF might contribute to activation of the intravascular coagulation cascade and thrombus growth on atherosclerotic lesions.

Recent studies in vitro indicate that, in addition to monocytes, various types of blood cells including neutrophils, eosinophils, and even platelets can synthesize TF. Although TF expression in these blood cells is a matter of debate, monocytes are probably the only blood cells that synthesize and express TF [53]. A related topic is the contribution of microparticles (MPs) to thrombus formation. Microparticles are small fragments of membrane-bound cytoplasm that are shed from the surface of activated or apoptotic cells [54]. The procoagulant activity of MPs increases with exposure to phosphatidylserine and TF. Clinical studies have found significantly elevated amounts of MPs in patients with acute coronary syndrome and ischemic stroke $[55,56]$. However, 

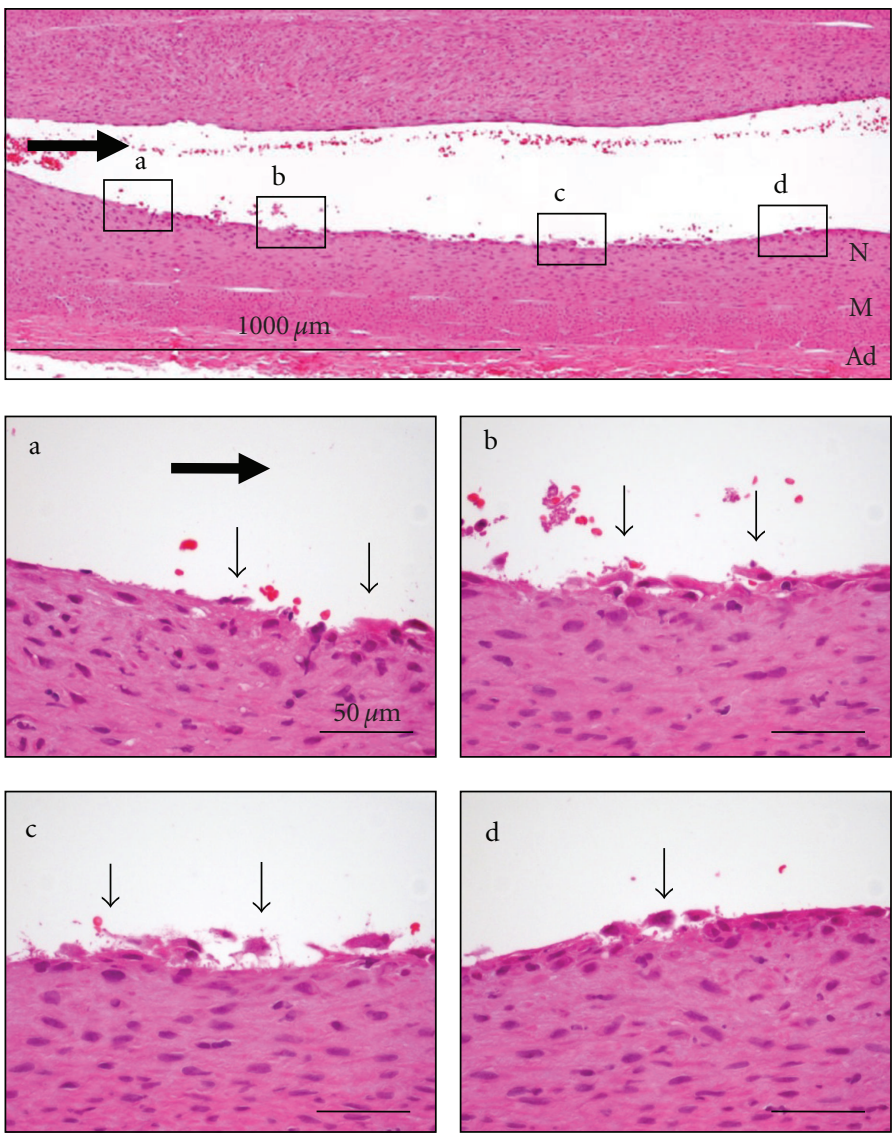

(a)
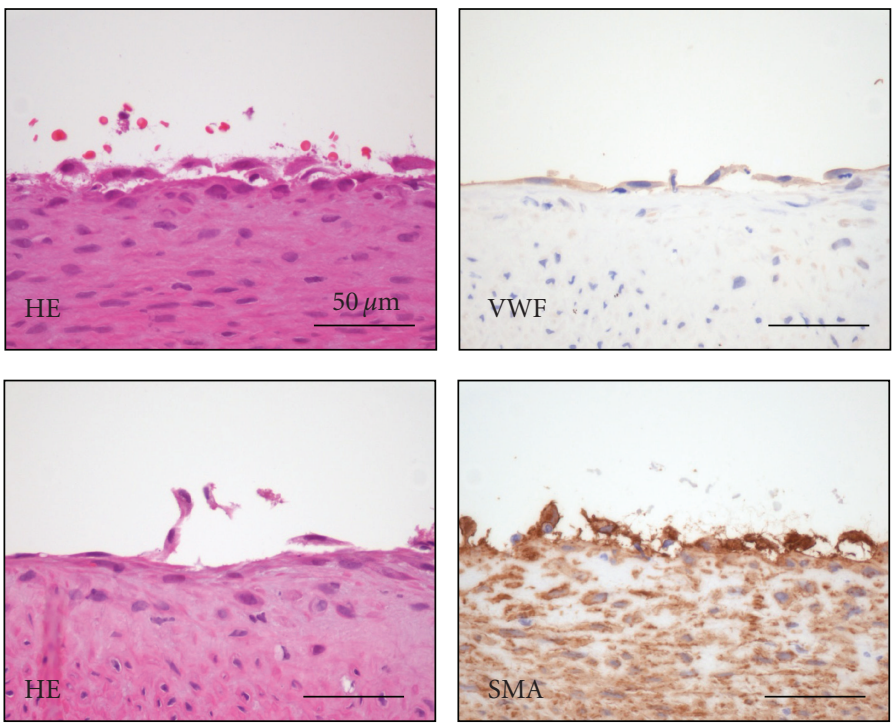

(b)

Figure 6: Representative longitudinal microphotographs of rabbit femoral artery with smooth muscle cell-rich neointima 15 minutes after vascular narrowing. (a) Broad superficial neointimal damage at poststenotic region. Neointimal cell detachment (small arrows) at high magnification. N, neointima; M, media; Ad, adventitia. Large arrow indicates blood flow. (b) Immunohistochemistry to detect von Willebrand factor (VWF, endothelial marker) and smooth muscle actin (SMA, smooth muscle marker) confirms detachment of endothelial and smooth muscle cells at poststenotic region. HE (hematoxylin eosin stain) (from Sumi et al. [45]). 


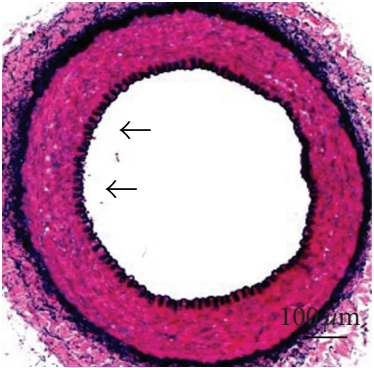

(a)

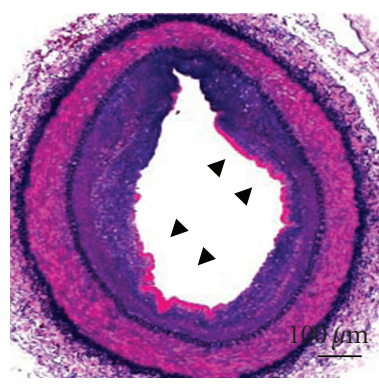

(e)

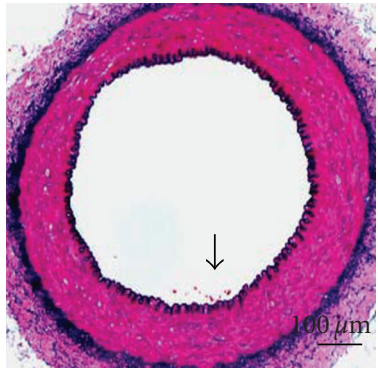

(b)

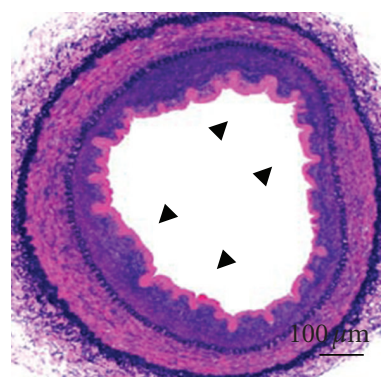

(f)

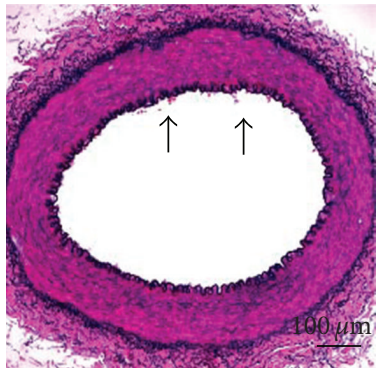

(c)

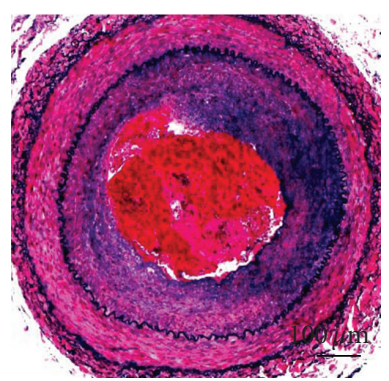

(g)

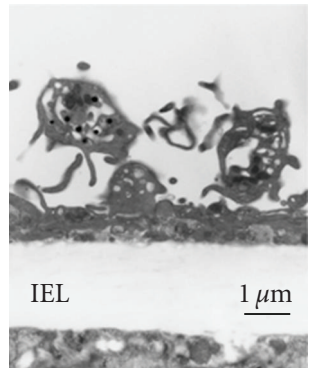

(d)

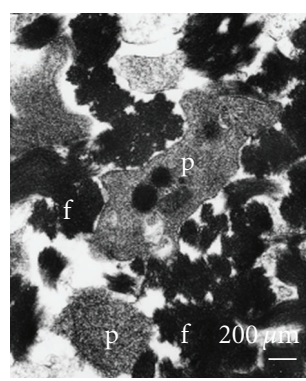

(h)

FIGURE 7: Mural thrombus growth on neointima after blood flow reduction. Small thrombi (arrows) are induced by balloon injury of normal femoral artery without (a) or with blood flow reduction to $50 \%$ (b) or $25 \%$ (c). Transmission electron micrograph (d) shows that thrombi consist of aggregated platelets (IEL, internal elastic lamina). Platelet fibrin-rich mural thrombi (arrow heads) are induced by balloon injury of neointima without (e) or with blood flow reduction to $50 \%$ (f). Neointimal injury with blood flow reduction to $25 \%$ causes occlusive thrombus formation $(\mathrm{g})$. Transmission electron micrograph (h) shows that these thrombi are composed of abundant fibrin strands (f) admixed with platelets (p). Hematoxylin eosin/Victria blue stain (a-c, e-g). (from Yamashita et al. [27] with permission).

whether elevated levels of MPs are a cause or a consequence of atherothrombosis remains unclear. Further studies are required to clarify the contribution of blood-derived TF and/or MPs to thrombus growth on atherosclerotic lesions.

Another mechanism that might contribute to thrombus growth in vivo is the intrinsic coagulation pathway that is initiated when coagulation factor XII (FXII) comes into contact with negatively charged surfaces. Factor XI (XI) is activated by activated FXII, thrombin, and activated XI. Feedback activation of FXI by thrombin promotes further thrombin generation in vitro [57]. Factor XI is present in platelet-fibrin thrombus-induced balloon injury of the rabbit atherosclerotic neointima, and anti-FXI antibody reduces thrombus growth without prolonging bleeding time [58]. This evidence indicates that intrinsic coagulation factors play a more significant role in thrombus growth than initial thrombus formation.

6.2. Role of Blood Flow on Thrombus Growth. Blood flow is a key modulator of thrombus growth. Some clinical studies have shown that blood flow is altered during coronary atherothrombosis. Marzilli et al. [59] identified an approximate $80 \%$ reduction in coronary blood flow during ischemia in patients with unstable angina. Hearts obtained from patients after sudden coronary death contained a mean of 4.5 intramyocardial microemboli, which are more common in plaque erosion than rupture and are associated with myocardial necrosis [60]. These mechanisms are considered to be largely responsible for the rapid elevation of distal vascular resistance due to microvascular embolism and/or vasoconstriction [61]. To assess how blood flow affects thrombus growth on atherosclerotic lesions, we reduced blood flow in rabbit femoral arteries after thrombus formation. Plateletfibrin mural thrombi on atherosclerotic neointima became occlusive in the setting of $>75 \%$ reduction in blood flow. In contrast, small platelet thrombi on normal arteries did not grow even under a 90\% reduction in blood flow (Figure 7) [27]. Thus, reduced blood flow during thrombus formation might affect only the formation of occlusive thrombus in atherosclerotic vessels.

In addition to distal vascular resistance, blood flow disturbed by acute vascular narrowing promotes thrombus growth at poststenotic regions. As described above, vascular narrowing of the rabbit femoral artery caused a superficial erosive injury at poststenotic regions of SMCrich neointima. Intimal damage significantly progressed at $3 \mathrm{~h}$ after vascular narrowing. Thrombi that developed on the neointima consisted of a mixture of aggregated platelets and a considerable amount of fibrin. In contrast, the identical vascular narrowing of normal femoral arteries also induced endothelial detachment with small platelet thrombi at poststenotic regions. Neither vascular narrowing nor antirabbit TF antibody affected whole blood hemostatic parameters in the rabbits, and no fibrin was generated in thrombi on eroded normal intima [45]. This evidence indicates that $\mathrm{TF}$ derived from eroded neointima plays a 


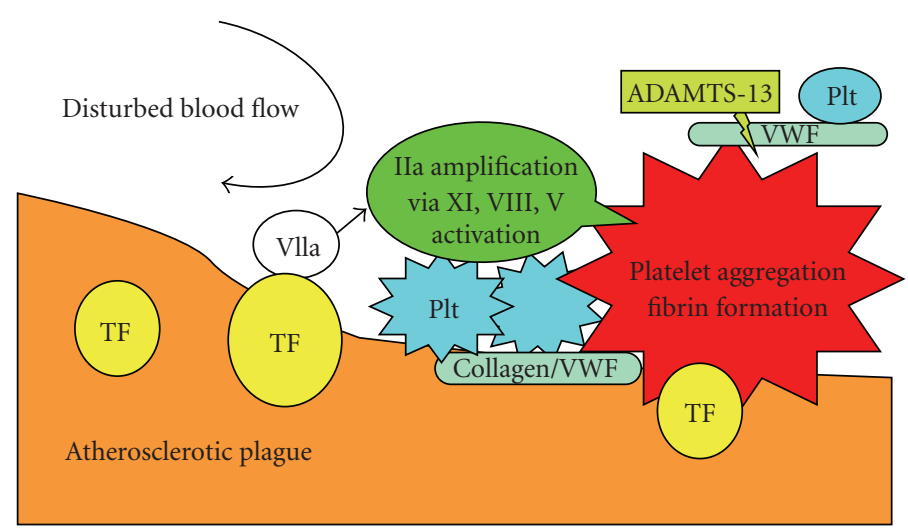

FIGURE 8: Schema of atherothrombus growth. Atherothrombus formation is initiated by plaque disruption and exposure of matrix proteins and tissue factor (TF). This induces initial platelet (Plt) adhesion/aggregation and activation of coagulation cascade. Thrombin (IIa) activates factor XI, factor VIII, and factor V on activated platelets, which amplify thrombin generation. Excess thrombin generation enhances fibrin formation and platelet aggregation on atherosclerotic plaque. The thrombin-dependent thrombus formation is mediated by plaque TF. Disturbed blood flow also promotes coagulation cascade and platelet recruitment by von Willebrand factor (VWF). The platelet recruitment is negatively regulated by ADAMTS-13 (a disintegrin and a metalloprotease with a thrombospondin type 1 motif 13).

crucial role in fibrin formation and thrombus growth rather than circulating TF. Therefore, flow that becomes disordered after plaque disruption might contribute to thrombus growth under conditions of distal resistance and of proximal stenosis.

The rheological effect on thrombus growth might be partly explained by a shear gradient-dependent platelet aggregation mechanism. Nesbitt et al. [62] showed using imaging modalities that a shear gradient-dependent platelet aggregation process is preceded by soluble agonistdependent aggregation stenotic microvessels in vitro and in vivo. A shear microgradient at poststenotic regions or at the downstream face of thrombi induces stable discoid platelet aggregates with restricted tethers, and the magnitude and spatial distribution of shear microgradients directly influence the size of platelet aggregates. This process required ligand binding to integrin $\alpha_{\mathrm{II}} \beta_{3}$ and an inositol triphosphate- and extracellular $\mathrm{Ca}^{2+}$-dependent transient $\mathrm{Ca}^{2+}$ flux, but not global platelet shape change, degranulation, or soluble agonists. Stabilized discoid platelet aggregates consolidated within the thrombus base and depended on soluble agonists. These findings suggest that platelets principally use a biomechanical platelet aggregation mechanism to promote the accumulation and stabilization of discoid platelets at sites of vascular injury. Vessel and/or thrombus geometry itself might promote thrombus formation.

6.3. Role of Platelet Recruitment on Thrombus Growth. Adhesion molecules and their receptors on platelets are essential for thrombus formation. The large, multimeric plasma protein VWF undergoes a conformational change when bound to a matrix, a process that allows VWF to bind GPIb $\alpha$. Recent experimental evidence in vitro and in vivo has shown that platelet recruitment on the thrombus surface is primarily mediated by VWF and GPIb $\alpha$ on flowing platelets $[63,64]$. Despite limited understanding of the roles of VWF on atherothrombus, its presence in human coronary thrombi suggests that it exerts an important function in this process $[5,6]$. A monoclonal antibody against the VWF A1 domain, which interacts with platelet GPIb $\alpha$ significantly inhibited the formation of fibrin-rich mural thrombus induced by balloon injury of the rabbit atherosclerotic neointima [65]. The antibody also completely inhibited occlusive thrombus formation on rabbit atherosclerotic vessels even when blood flow was disturbed [27]. Thus, VWF plays an important role in thrombus propagation on atherosclerotic lesions via platelet recruitment.

The size of the VWF multimer can affect that of thrombus, and the size of the VWF multimer is regulated by a plasma protease, a disintegrin, and a metalloprotease with a thrombospondin type 1 motif 13 (ADAMTS-13). A deficiency in ADAMTS-13 activity leads to an increased level of circulating ultralarge VWF multimers, and this correlates with the onset of general thrombotic thrombocytopenic purpura (TTP). Clinical evidence suggests that the size of the VWF multimer is dysregulated in patients with AMI. The ratio of VWF/ADAMTS-13 antigen is higher in patients with AMI than with stable angina pectoris, and the correlation between plasma VWF antigen and ADAMTS-13 activity is inverse in patients with AMI [66]. ADAMTS-13 closely localizes with VWF in fresh coronary thrombi from AMI patients [67]. Reducing ADAMTS-13 activity using a monoclonal antibody against the disintegrin-like domain enhances platelet thrombus growth on immobilized type I collagen at a high shear rate and platelet-fibrin thrombus formation on the injured neointima of rabbit femoral arteries [67]. This study also showed that large VWF multimers are cleaved during platelet thrombus formation under a high shear rate. The site of VWF cleavage by ADAMTS-13 is localized to the surface of platelet thrombus, and ADAMTS- 13 activity is shear dependent [68]. Thus, ADAMTS-13 might function at sites of ongoing thrombus generation and limit thrombus growth. 


\section{Limitations and Future Directions}

The rabbit model of atherothrombosis has several limitations. The histological features of unstable plaque include a high lipid content, intensive macrophage accumulation, a large necrotic core with a thin fibrous cap, and few SMCs. Although a high-cholesterol diet combined with balloon injury induces macrophage-rich lesions at damaged sites, a large necrotic core with a thin fibrous cap is difficult to develop. This limits assessments of the pathogenesis of plaque rupture in this model. The thickness $(\sim 500 \mu \mathrm{m})$ of diseased arterial walls interferes with intravital microscopic visualization of thrombus formation in vivo, and the rabbit is at a distinct disadvantage compared with the mouse in terms of genomic and protein information.

Despite these potential limitations, the model based on human pathology will nevertheless provide valuable information about the pathophysiology of atherothrombosis. Larger size in rabbits will be more suitable than mice for stent implantation as well as direct vascular or thrombus imaging using magnetic resonance imaging and positron emission tomography $[69,70]$. Accumulating genomic information about rabbits is already publicly available. Important findings have been generated from studies of atherogenesis and lipid metabolism in transgenic rabbits generated by pronuclear microinjection [71]. Several embryonic stem cell lines have major important features in common with human embryonic stem cells $[72,73]$. Stable pluripotent stem cells have recently been generated from rabbit somatic cells by the introduction of four human transcription factors [74]. The molecular mechanisms of atherothrombosis will soon be uncovered via the rapid accumulation of molecular information and progress in stem cell technology.

\section{Conclusions}

Procoagulant activity is increased by active TF expression in human atherosclerotic lesions, and occlusive atherothrombus consistently comprises aggregated platelets, fibrin, and erythrocytes. Balloon injury of the neointima expressing TF induces large plate-fibrin thrombi in diseased arteries of a rabbit model. Enhanced platelet aggregation and fibrin formation in atherosclerotic arteries is primarily mediated by neointimal TF in this model. Studies using this model have revealed thrombin-dependent thrombus formation in atherosclerotic vessels, plaque erosion induced by disturbed blood flow, thrombogenic vasoconstriction of SMC-rich neointima, and the factors involved in thrombus growth (Figure 8). Overcoming the limitations of the rabbit model using emerging transgenic and stem cell technologies might lead to its establishment as an invaluable experimental tool for understanding the mechanisms of atherosclerosis.

\section{Acknowledgment}

This study was supported in part by Grants-in-Aid for Scientific Research in Japan (nos. 19790293, 20390102, and 21590374) from the Ministry of Education, Science, Sports and Culture of Japan.

\section{References}

[1] M. J. Davies and A. Thomas, "Thrombosis and acute coronary-artery lesions in sudden cardiac ischemic death," New England Journal of Medicine, vol. 310, no. 18, pp. 11371140, 1984.

[2] T. A. Drake, J. H. Morissey, and T. S. Edgington, "Selective cellular expression of tissue factor in human tissues. Implications for disorders of hemostasis and thrombosis," American Journal of Pathology, vol. 134, no. 5, pp. 1087-1097, 1989.

[3] M. J. Chapman, "Animal lipoproteins: chemistry, structure, and comparative aspects," Journal of Lipid Research, vol. 21, no. 7, pp. 789-853, 1980.

[4] Y. Sato, K. Hatakeyama, A. Yamashita, K. Marutsuka, A. Sumiyoshi, and Y. Asada, "Proportion of fibrin and platelets differs in thrombi on ruptured and eroded coronary atherosclerotic plaques in humans," Heart, vol. 91, no. 4, pp. 526-530, 2005.

[5] A. Yamashita, T. Sumi, S. Goto et al., "Detection of von Willebrand factor and tissue factor in platelets-fibrin rich coronary thrombi in acute myocardial infarction," American Journal of Cardiology, vol. 97, no. 1, pp. 26-28, 2006.

[6] K. Nishihira, A. Yamashita, T. Ishikawa, K. Hatakeyama, Y. Shibata, and Y. Asada, "Composition of thrombi in late drugeluting stent thrombosis versus de novo acute myocardial infarction," Thrombosis Research, vol. 125, pp. 464-470, 2010.

[7] J. N. Wilcox, K. M. Smith, S. M. Schwartz, and D. Gordon, "Localization of tissue factor in the normal vessel wall and in the atherosclerotic plaque," Proceedings of the National Academy of Sciences of the United States of America, vol. 86, no. 8, pp. 2839-2843, 1989.

[8] K. Hatakeyama, Y. Asada, K. Marutsuka, Y. Sato, Y. Kamikubo, and A. Sumiyoshi, "Localization and activity of tissue factor in human aortic atherosclerotic lesions," Atherosclerosis, vol. 133, no. 2, pp. 213-219, 1997.

[9] R. Virmani, F. D. Kolodgie, A. P. Burke, A. Farb, and S. M. Schwartz, "Lessons from sudden coronary death: a comprehensive morphological classification scheme for atherosclerotic lesions," Arteriosclerosis, Thrombosis, and Vascular Biology, vol. 20, no. 5, pp. 1262-1275, 2000.

[10] J. I. Kotani, G. S. Mintz, M. T. Castagna et al., "Intravascular ultrasound analysis of infarct-related and non-infarct-related arteries in patients who presented with an acute myocardial infarction," Circulation, vol. 107, no. 23, pp. 2889-2893, 2003.

[11] A. P. Burke, F. D. Kolodgie, A. Farb et al., "Healed plaque ruptures and sudden coronary death: evidence that subclinical rupture has a role in plaque progression," Circulation, vol. 103, no. 7, pp. 934-940, 2001.

[12] Y. Sato, K. Hatakeyama, K. Marutsuka, and Y. Asada, "Incidence of asymptomatic coronary thrombosis and plaque disruption: comparison of non-cardiac and cardiac deaths among autopsy cases," Thrombosis Research, vol. 124, no. 1, pp. 19-23, 2009.

[13] P. K. Shah, "Mechanisms of plaque vulnerability and rupture," Journal of the American College of Cardiology, vol. 41, no. 4, pp. 15S-22S, 2003.

[14] K. Nishihira, T. Imamura, K. Hatakeyama et al., "Expression of interleukin-18 in coronary plaque obtained by atherectomy from patients with stable and unstable angina," Thrombosis Research, vol. 121, no. 2, pp. 275-279, 2007.

[15] K. Nishihira, T. Imamura, A. Yamashita et al., "Increased expression of interleukin-10 in unstable plaque obtained by directional coronary atherectomy," European Heart Journal, vol. 27, no. 14, pp. 1685-1689, 2006. 
[16] S. W. Waldo, Y. Li, C. Buono et al., "Heterogeneity of human macrophages in culture and in atherosclerotic plaques," American Journal of Pathology, vol. 172, no. 4, pp. 1112-1126, 2008.

[17] F. D. Kolodgie, H. K. Gold, A. P. Burke et al., "Intraplaque hemorrhage and progression of coronary atheroma," New England Journal of Medicine, vol. 349, no. 24, pp. 2316-2325, 2003.

[18] K. Nishihira, A. Yamashita, T. Imamura et al., "Thioredoxin in coronary culprit lesions: possible relationship to oxidative stress and intraplaque hemorrhage," Atherosclerosis, vol. 201, no. 2, pp. 360-367, 2008.

[19] P. Golino, F. Piscione, J. T. Willerson et al., "Divergent effects of serotonin on coronary-artery dimensions and blood flow in patients with coronary atherosclerosis and control patients," New England Journal of Medicine, vol. 324, no. 10, pp. 641648, 1991.

[20] E. Durand, A. Scoazec, A. Lafont et al., "In vivo induction of endothelial apoptosis leads to vessel thrombosis and endothelial denudation: a clue to the understanding of the mechanisms of thrombotic plaque erosion," Circulation, vol. 109, no. 21, pp. 2503-2506, 2004.

[21] K. D. Kurz, B. W. Main, and G. E. Sandusky, "Rat model of arterial thrombosis induced by ferric chloride," Thrombosis Research, vol. 60, no. 4, pp. 269-280, 1990.

[22] H. Matsuno, T. Uematsu, S. Nagashima, and M. Nakashima, "Photochemically induced thrombosis model in rat femoral artery and evaluation of effects of heparin and tissue-type plasminogen activator with use of this model," Journal of Pharmacological Methods, vol. 25, no. 4, pp. 303-317, 1991.

[23] A. R. Saniabadi, K. Umemura, N. Matsumoto, S. Sakuma, and M. Nakashima, "Vessel wall injury and arterial thrombosis induced by a photochemical reaction," Thrombosis and Haemostasis, vol. 73, no. 5, pp. 868-872, 1995.

[24] J. Inamo, E. Belougne, and C. Doutremepuich, "Importance of photo activation of Rose Bengal for platelet activation in experimental models of photochemically induced thrombosis," Thrombosis Research, vol. 83, no. 3, pp. 229-235, 1996.

[25] E. Furukoji, M. Matsumoto, A. Yamashita et al., "Adenovirusmediated transfer of human placental ectonucleoside triphosphate diphosphohydrolase to vascular smooth muscle cells suppresses platelet aggregation in vitro and arterial thrombus formation in vivo," Circulation, vol. 111, no. 6, pp. 808-815, 2005.

[26] K. Hatakeyama, Y. Asada, K. Marutsuka, H. Kataoka, Y. Sato, and A. Sumiyoshi, "Expression of tissue factor in the rabbit aorta after balloon injury," Atherosclerosis, vol. 139, no. 2, pp. 265-271, 1998.

[27] A. Yamashita, E. Furukoji, K. Marutsuka et al., "Increased vascular wall thrombogenicity combined with reduced blood flow promotes occlusive thrombus formation in rabbit femoral artery," Arteriosclerosis, Thrombosis, and Vascular Biology, vol. 24, no. 12, pp. 2420-2424, 2004.

[28] A. Yamashita, S. Matsuda, T. Matsumoto et al., "Thrombin generation by intimal tissue factor contributes to thrombus formation on macrophage-rich neointima but not normal intima of hyperlipidemic rabbits," Atherosclerosis, vol. 206, no. 2, pp. 418-426, 2009.

[29] K. Kugiyama, T. Murohara, H. Yasue et al., "Increased constrictor response to acetylcholine of the isolated coronary arteries from patients with variant angina," International Journal of Cardiology, vol. 52, no. 3, pp. 223-233, 1995.
[30] A. Farb, A. P. Burke, A. L. Tang et al., "Coronary plaque erosion without rupture into a lipid core: a frequent cause of coronary thrombosis in sudden coronary death," Circulation, vol. 93, no. 7, pp. 1354-1363, 1996.

[31] A. Yamashita, K. Shoji, T. Tsuruda et al., "Medial and adventitial macrophages are associated with expansive atherosclerotic remodeling in rabbit femoral artery," Histology and Histopathology, vol. 23, no. 2, pp. 127-136, 2008.

[32] J. T. B. Crawley, S. Zanardelli, C. K. N. K. Chion, and D. A. Lane, "The central role of thrombin in hemostasis," Journal of Thrombosis and Haemostasis, vol. 5, no. 1, pp. 95-101, 2007.

[33] L. Chi, G. Gibson, Y. W. Peng et al., "Characterization of a tissue factor/factor VIIa-dependent model of thrombosis in hypercholesterolemic rabbits," Journal of Thrombosis and Haemostasis, vol. 2, no. 1, pp. 85-92, 2004.

[34] Y. Nakashima, A. S. Plump, E. W. Raines, J. L. Breslow, and R. Ross, "ApoE-deficient mice develop lesions of all phases of atherosclerosis throughout the arterial tree," Arteriosclerosis and Thrombosis, vol. 14, no. 1, pp. 133-140, 1994.

[35] D. T. Eitzman, R. J. Westrick, Z. Xu, J. Tyson, and D. Ginsburg, "Hyperlipidemia promotes thrombosis after injury to atherosclerotic vessels in apolipoprotein E-deficient mice," Arteriosclerosis, Thrombosis, and Vascular Biology, vol. 20, no. 7, pp. 1831-1834, 2000.

[36] M. J. E. Kuijpers, K. Gilio, S. Reitsma et al., "Complementary roles of platelets and coagulation in thrombus formation on plaques acutely ruptured by targeted ultrasound treatment: a novel intravital model," Journal of Thrombosis and Haemostasis, vol. 7, no. 1, pp. 152-161, 2009.

[37] P. Constantinides and R. N. Chakravarti, "Rabbit arterial thrombosis production by systemic procedures," Archives of Pathology, vol. 72, pp. 197-208, 1961.

[38] G. S. Abela, P. D. Picon, S. E. Friedl et al., "Triggering of plaque disruption and arterial thrombosis in an atherosclerotic rabbit model," Circulation, vol. 91, no. 3, pp. 776-784, 1995.

[39] M. T. Johnstone, R. M. Botnar, A. S. Perez et al., "In vivo magnetic resonance imaging of experimental thrombosis in a rabbit model," Arteriosclerosis, Thrombosis, and Vascular Biology, vol. 21, no. 9, pp. 1556-1560, 2001.

[40] K. Aziz, K. Berger, K. Claycombe, R. Huang, R. Patel, and G. S. Abela, "Noninvasive detection and localization of vulnerable plaque and arterial thrombosis with computed tomography angiography/positron emission tomography," Circulation, vol. 117, no. 16, pp. 2061-2070, 2008.

[41] Y. Miyao, K. Kugiyama, H. Kawano et al., "Diffuse intimal thickening of coronary arteries in patients with coronary spastic angina," Journal of the American College of Cardiology, vol. 36, no. 2, pp. 432-437, 2000.

[42] H. Hao, G. Gabbiani, E. Camenzind, M. Bacchetta, R. Virmani, and M. L. Bochaton-Piallat, "Phenotypic modulation of intima and media smooth muscle cells in fatal cases of coronary artery lesion," Arteriosclerosis, Thrombosis, and Vascular Biology, vol. 26, no. 2, pp. 326-332, 2006.

[43] O. Tricot, Z. Mallat, C. Heymes, J. Belmin, G. Lesèche, and A. Tedgui, "Relation between endothelial cell apoptosis and blood flow direction in human atherosclerotic plaques," Circulation, vol. 101, no. 21, pp. 2450-2453, 2000.

[44] D. L. Fry, "Acute vascular endothelial changes associated with increased blood velocity gradients," Circulation Research, vol. 22, no. 2, pp. 165-197, 1968. 
[45] T. Sumi, A. Yamashita, S. Matsuda et al., "Disturbed blood flow induces erosive injury to smooth muscle cell-rich neointima and promotes thrombus formation in rabbit femoral arteries," Journal of Thrombosis and Haemostasis, vol. 8, no. 6, pp. 1394-1402, 2010.

[46] S. Oshima, H. Yasue, H. Ogawa, K. Okumura, and K. Matsuyama, "Fibrinopeptide A is released into the coronary circulation after coronary spams," Circulation, vol. 82, no. 6, pp. 2222-2225, 1990.

[47] H. Shimokawa, "Cellular and molecular mechanisms of coronary artery spasm: lessons from animal models," Japanese Circulation Journal, vol. 64, no. 1, pp. 1-12, 2000.

[48] K. Nishihira, A. Yamashita, N. Tanaka et al., "Inhibition of 5hydroxytryptamine receptor prevents occlusive thrombus formation on neointima of the rabbit femoral artery," Journal of Thrombosis and Haemostasis, vol. 4, no. 1, pp. 247-255, 2006.

[49] K. Nishihira, A. Yamashita, N. Tanaka et al., "Serotonin induces vasoconstriction of smooth muscle cell-rich neointima through 5-hydroxytryptamine2A receptor in rabbit femoral arteries," Journal of Thrombosis and Haemostasis, vol. 6, no. 7, pp. 1207-1214, 2008.

[50] N. Mackman, "Role of tissue factor in hemostasis, thrombosis, and vascular development," Arteriosclerosis, Thrombosis, and Vascular Biology, vol. 24, no. 6, pp. 1015-1022, 2004.

[51] J. Chou, N. Mackman, G. Merrill-Skoloff, B. Pedersen, B. C. Furie, and B. Furie, "Hematopoietic cell-derived microparticle tissue factor contributes to fibrin formation during thrombus propagation," Blood, vol. 104, no. 10, pp. 3190-3197, 2004.

[52] S. M. Day, J. L. Reeve, B. Pedersen et al., "Macrovascular thrombosis is driven by tissue factor derived primarily from the blood vessel wall," Blood, vol. 105, no. 1, pp. 192-198, 2005.

[53] B. Østerud, "Tissue factor expression in blood cells," Thrombosis Research, vol. 125, no. 1, pp. S31-S34, 2010.

[54] A. Blann, E. Shantsila, and A. Shantsila, "Microparticles and arterial disease," Seminars in Thrombosis and Hemostasis, vol. 35, no. 5, pp. 488-496, 2009.

[55] N. Singh, C. H. Gemmell, P. A. Daly, and E. L. Yeo, "Elevated platelet-derived microparticle levels during unstable angina," Canadian Journal of Cardiology, vol. 11, no. 11, pp. 1015-1021, 1995.

[56] T. Geiser, M. Sturzenegger, U. Genewein, A. Haeberli, and J. H. Beer, "Mechanisms of cerebrovascular events as assessed by procoagulant activity, cerebral microemboli, and platelet microparticles in patients with prosthetic heart valves," Stroke, vol. 29, no. 9, pp. 1770-1777, 1998.

[57] D. Gailani and G. J. Broze, "Factor XI activation in a revised model of blood coagulation," Science, vol. 253, no. 5022, pp. 909-912, 1991.

[58] A. Yamashita, K. Nishihira, T. Kitazawa et al., "Factor XI contributes to thrombus propagation on injured neointima of the rabbit iliac artery," Journal of Thrombosis and Haemostasis, vol. 4, no. 7, pp. 1496-1501, 2006.

[59] M. Marzilli, G. Sambuceti, S. Fedele, and A. L'Abbate, "Coronary microcirculatory vasoconstriction during ischemia in patients with unstable angina," Journal of the American College of Cardiology, vol. 35, no. 2, pp. 327-334, 2000.

[60] R. S. Schwartz, A. Burke, A. Farb et al., "Microemboli and microvascular obstruction in acute coronary thrombosis and sudden coronary death: relation to epicardial plaque histopathology," Journal of the American College of Cardiology, vol. 54, no. 23, pp. 2167-2173, 2009.

[61] R. Erbel and G. Heusch, "Coronary microembolization," Journal of the American College of Cardiology, vol. 36, no. 1, pp. 22-24, 2000.
[62] W. S. Nesbitt, E. Westein, F. J. Tovar-Lopez et al., "A shear gradient-dependent platelet aggregation mechanism drives thrombus formation," Nature Medicine, vol. 15, no. 6, pp. 665-673, 2009.

[63] S. Kulkarni, S. M. Dopheide, C. L. Yap et al., "A revised model of platelet aggregation," Journal of Clinical Investigation, vol. 105, no. 6, pp. 783-791, 2000.

[64] W. Bergmeier, C. L. Piffath, T. Goerge et al., "The role of platelet adhesion receptor GPIb $\alpha$ far exceeds that of its main ligand, von Willebrand factor, in arterial thrombosis," Proceedings of the National Academy of Sciences of the United States of America, vol. 103, no. 45, pp. 16900-16905, 2006.

[65] A. Yamashita, Y. Asada, H. Sugimura et al., "Contribution of von Willebrand factor to thrombus formation on neointima of rabbit stenotic iliac artery under high blood-flow velocity," Arteriosclerosis, Thrombosis, and Vascular Biology, vol. 23, no. 6, pp. 1105-1110, 2003.

[66] K. Kaikita, K. Soejima, M. Matsukawa, T. Nakagaki, and H. Ogawa, "Reduced von Willebrand factor-cleaving protease (ADAMTS13) activity in acute myocardial infarction," Journal of Thrombosis and Haemostasis, vol. 4, no. 11, pp. 2490-2493, 2006.

[67] S. Moriguchi-Goto, A. Yamashita, N. Tamura et al., "ADAMTS-13 attenuates thrombus formation on type I collagen surface and disrupted plaques under flow conditions," Atherosclerosis, vol. 203, no. 2, pp. 409-416, 2009.

[68] Y. Shida, K. Nishio, M. Sugimoto et al., "Functional imaging of shear-dependent activity of ADAMTS13 in regulating mural thrombus growth under whole blood flow conditions," Blood, vol. 111, no. 3, pp. 1295-1298, 2008.

[69] Y. Kuroiwa, A. Yamashita, T. Miyati et al., "Atherosclerotic lesions rich in macrophages or smooth muscle cells discriminated in rabbit iliac arteries based on T1 relaxation time and lipid content," Academic Radiology, vol. 17, no. 2, pp. 230-238, 2010.

[70] R. Kawamoto, A. Yamashita, K. Nishihira et al., "Different inflammatory response and oxidative stress in neointimal hyperplasia after balloon angioplasty and stent implantation in cholesterol-fed rabbits," Pathology Research and Practice, vol. 202, no. 6, pp. 447-456, 2006.

[71] J. Fan and T. Watanabe, "Transgenic rabbits as therapeutic protein bioreactors and human disease models," Pharmacology and Therapeutics, vol. 99, no. 3, pp. 261-282, 2003.

[72] Z. F. Fang, H. Gai, Y. Z. Huang et al., "Rabbit embryonic stem cell lines derived from fertilized, parthenogenetic or somatic cell nuclear transfer embryos," Experimental Cell Research, vol. 312, no. 18, pp. 3669-3682, 2006.

[73] A. Honda, M. Hirose, K. Inoue et al., "Stable embryonic stem cell lines in rabbits: potential small animal models for human research," Reproductive BioMedicine Online, vol. 17, no. 5, pp. 706-715, 2008.

[74] A. Honda, M. Hirose, M. Hatori et al., "Generation of induced pluripotent stem cells in rabbits: potential experimental models for human regenerative medicine," Journal of Biological Chemistry, vol. 285, no. 41, pp. 31362-31369, 2010. 


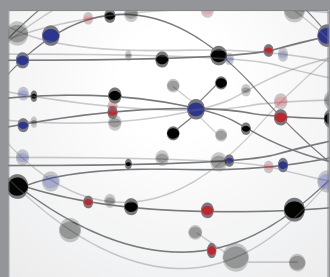

The Scientific World Journal
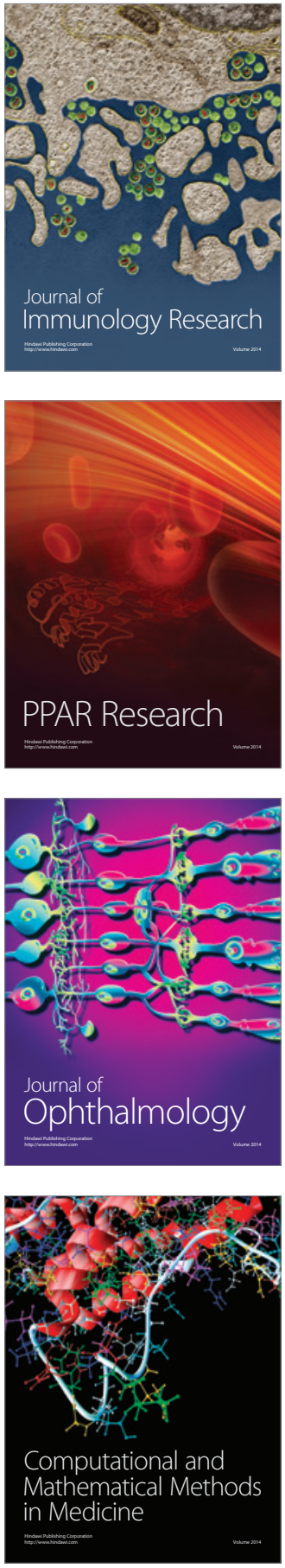

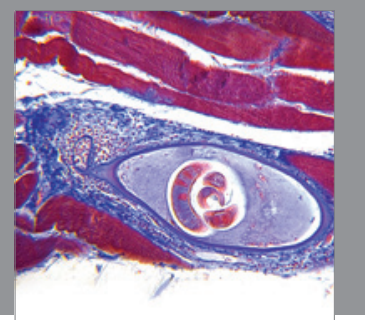

Gastroenterology

Research and Practice
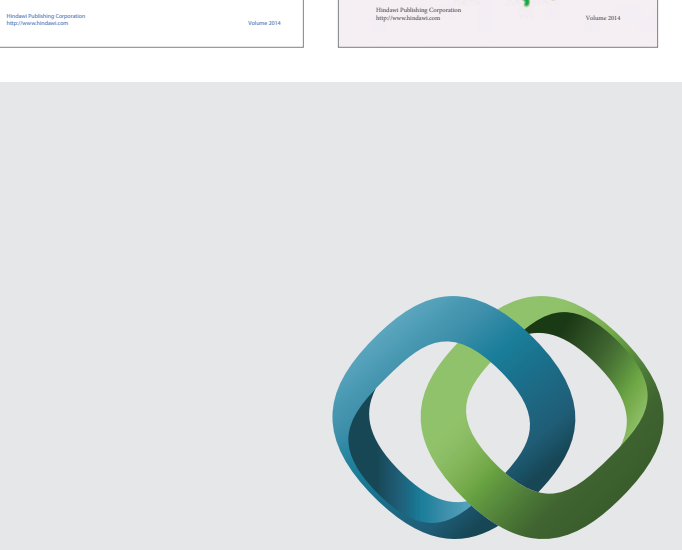

\section{Hindawi}

Submit your manuscripts at

http://www.hindawi.com
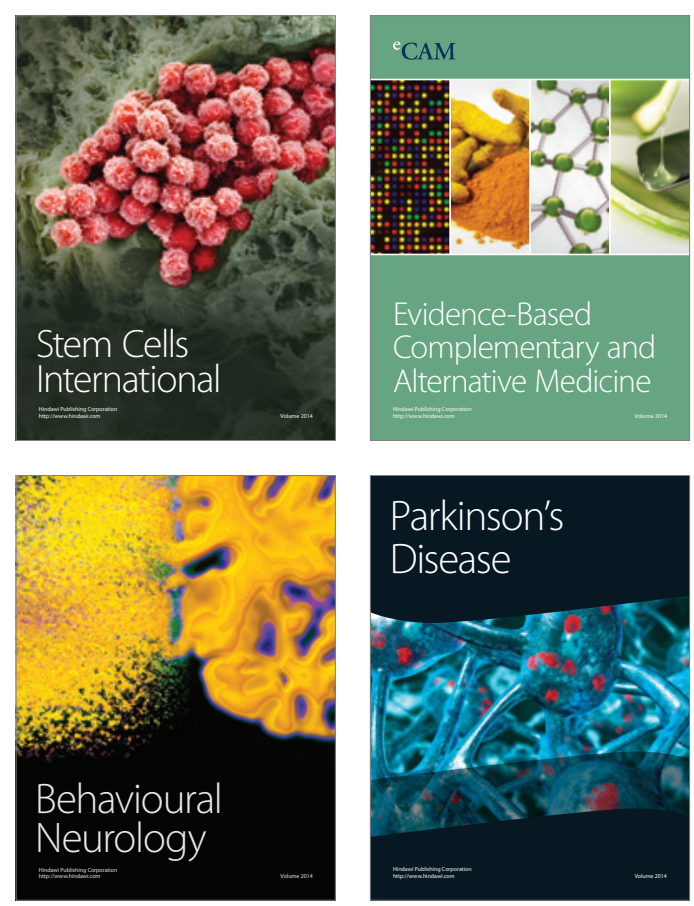

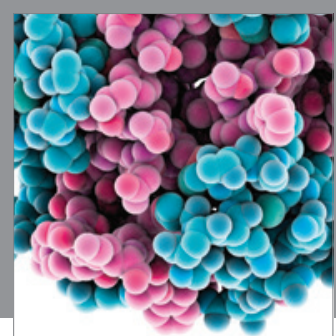

Journal of
Diabetes Research

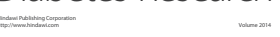

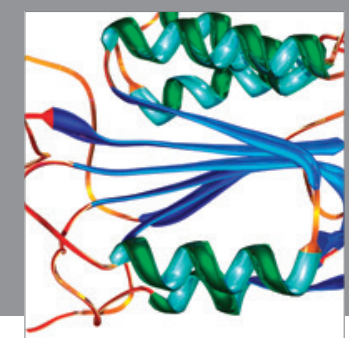

Disease Markers
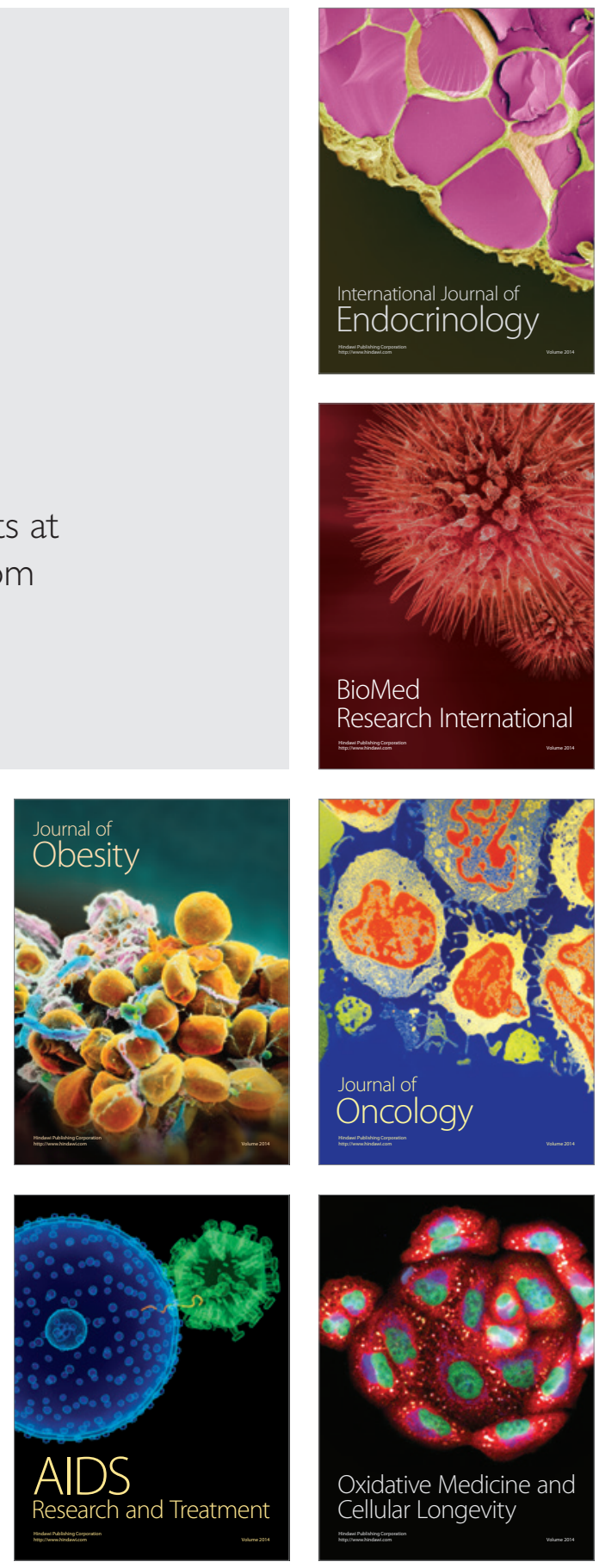